\title{
ON THE STABILITY OF A PERTURBED NONLINEAR SYSTEM
}

AARON STRAUSS

Consider the systems of ordinary differential equations

$$
\begin{aligned}
y^{\prime} & =A(t) y, \quad\left(\prime=\frac{d}{d t}\right), \\
x^{\prime} & =A(t) x+g(t, x), \\
y^{\prime} & =f(t, y), \\
x^{\prime} & =f(t, x)+g(t, x),
\end{aligned}
$$

where each system has a right-hand side continuous on $D=\{(t, x)$ : $\left.t \geqq 0, x \in E^{n}\right\}$ and sufficiently smooth there for the uniqueness of all solutions. For $x \in E^{n}$, let $|x|=\left|x_{1}\right|+\cdots+\left|x_{n}\right|$. The solution of (1) through some point $\left(t_{0}, x_{0}\right) \in D$ will be denoted by $y\left(t, t_{0}, x_{0}\right)$; the solution of (2) by $x\left(t, t_{0}, x_{0}\right)$. A solution $y(t)$ of (1) exists on some maximal interval $[0, \beta)$ or $(\alpha, \beta)$, where $0 \leqq \alpha<\beta \leqq+\infty$, and is said to be

bounded if it exists and is bounded on $[0, \infty)$,

bounded in the future if $\beta=+\infty$ and if for every $\gamma>\alpha, y(t)$ is bounded on $[\gamma, \infty)$,

stable if $\beta=+\infty$ and if for every $t_{0}>\alpha$ (or $t_{0} \geqq 0$ ) and every $\epsilon>0$, there exists $\delta\left(\epsilon, t_{0}\right)>0$ such that for all $\bar{y}_{0} \in E^{n}$ satisfying $\left|\bar{y}_{0}-y\left(t_{0}\right)\right|<\delta$, we have $\left|y\left(t, t_{0}, \bar{y}_{0}\right)-y(t)\right|<\epsilon$, for all $t \geqq t_{0}$,

uniformly stable if it is stable and $\delta$ is independent of $t_{0}$.

Notice that $y(t)$ can be uniformly stable and yet not exist on $[0, \infty)$. If $y(t)$ does exist on $[0, \infty)$, then uniform stability and stability are different concepts, but boundedness and boundedness in the future are equivalent.

Consider the following hypotheses:

$\mathrm{H}_{1}^{\circ}: A(t)$ is continuous on $[0, \infty)$.

$\mathrm{H}_{1}$ : The Jacobian matrix $f_{y}(t, y)$ is continuous on $D$.

$\mathrm{H}_{2}^{\circ}$ : All solutions of $\left(1^{\circ}\right)$ are uniformly stable.

$\mathrm{H}_{2}$ : All solutions of (1) are uniformly stable, at least one is bounded, and for every $M>0$, there exists $N(M)>0$ such that if $|z| \leqq M$, then $\left|R\left(t, t_{0}, z\right)\right| \leqq N$ for all $t \geqq t_{0} \geqq 0$, where $R\left(t, t_{0}, z\right)$ is the Jacobian matrix $\left(\partial y_{j} /\left(t, t_{0}, z\right) \partial z_{i}\right)$ of partial derivatives of the solution $y\left(t, t_{0}, z\right)$ of (1).

$\mathrm{H}_{3}$ : For every $M>0$, there exists a continuous function $h_{M}(t) \geqq 0$

Received by the editors October 12, 1965. 
such that if $t \geqq 0$ and $|x| \leqq M$, then $|g(t, x)| \leqq h_{M}(t)$, where $\int_{0}^{\infty} h_{M}(t) d t$ $<\infty$.

Onuchic [1] recently proved

Theorem $1^{\circ}$. Let $\left(1^{\circ}\right)$ satisfy $\mathrm{H}_{1}^{\circ}$ and $\mathrm{H}_{2}^{\circ}$. Let $g(t, x)$ satisfy $\mathrm{H}_{3}$. Then (a) for every solution $y(t)$ of $\left(1^{\circ}\right)$, there exist $T \geqq 0$ and $\delta>0$ such that if $t_{0} \geqq T$ and $\left|y\left(t_{0}\right)-x_{0}\right|<\delta$, then the solution $x\left(t, t_{0}, x_{0}\right)$ of $\left(2^{\circ}\right)$ is bounded in the future; and (b) every solution of $\left(2^{\circ}\right)$ which is bounded in the future is stable.

It is the purpose of this note to generalize Onuchic's theorem to perturbed nonlinear systems.

THEOREM 1. Let (1) satisfy $\mathrm{H}_{1}$ and $\mathrm{H}_{2}$. Let $g(t, x)$ satisfy $\mathrm{H}_{3}$. Then (a) and (b) hold for (1) and (2).

Before proving Theorem 1 we make several remarks.

1. If $f(t, y)=A(t) y$, then $R\left(t, t_{0}, z\right)=Y(t) Y^{-1}\left(t_{0}\right)$, where $Y(t)$ is a fundamental matrix for $\left(1^{\circ}\right)$. In this case, $\mathrm{H}_{2}^{\circ}$ holds if and only if there exists a constant $K>0$ such that $\left|Y(t) Y^{-1}\left(t_{0}\right)\right| \leqq K$ for all $t \geqq t_{0} \geqq 0$ (see $[2$, p. 45]). Furthermore, the zero solution is certainly bounded. Thus $\mathrm{H}_{2}^{\circ}$ and $\mathrm{H}_{2}$ are equivalent in the linear case. Clearly, $\mathrm{H}_{1}^{\circ}$ and $\mathrm{H}_{1}$ are equivalent, also. Hence Theorem $1^{\circ}$ is a special case of Theorem 1.

2. Onuchic used the Tychonoff fixed point theorem to prove Theorem $1^{\circ}$. Thus, for the linear case, our proof gives a new proof of Onuchic's result.

3. In general, $\mathrm{H}_{2}$ implies that all solutions of (1) are bounded in the future. To see this, consider any $t_{0} \geqq 0$. Define

$$
B=\left\{y_{0} \in E^{n}: y\left(t, t_{0}, y_{0}\right) \text { is bounded in the future }\right\} .
$$

The existence of a bounded solution of (1) implies that $B$ is not empty, while the stability of all solutions shows that both $B$ and its complement are open sets. Since $E^{n}$ is connected, $B=E^{n}$.

4. If we omit the statement "at least one solution is bounded" from $\mathrm{H}_{2}$, Theorem 1 may become false, as can be seen by the nonlinear example

$$
\begin{aligned}
& y^{\prime}=1, \\
& x^{\prime}=1+0,
\end{aligned}
$$

where $R\left(t, t_{0}, z\right) \equiv 1$, all solutions of (3) are uniformly stable, but none are bounded. Here, (a) is false and (b) is vacuous. 
5. An example of a nonlinear system satisfying $\mathrm{H}_{2}$ is the first order equation

$$
y^{\prime}=-\frac{1}{2} y^{3}
$$

with solution $y\left(t, t_{0}, y_{0}\right)=y_{0}\left[y_{0}^{2}\left(t-t_{0}\right)+1\right]^{-(1 / 2)}$, so that $R\left(t, t_{0}, z\right)$ $=\left[z^{2}\left(t-t_{0}\right)+1\right]^{-(3 / 2)}$. Hence $\left|R\left(t, t_{0}, z\right)\right| \leqq 1$ for all $t \geqq t_{0} \geqq 0$ and all real $z$. However, it would be nice to characterize those nonlinear systems for which $\mathrm{H}_{2}$ holds. This remains an open problem.

6. The following example shows that (a) in Theorem 1 is the best possible general result concerning the existence of solutions of (2) which are bounded in the future. Consider the first order equations

$$
\begin{aligned}
& y^{\prime}=0, \\
& x^{\prime}=0+e^{-2 t} x^{3},
\end{aligned}
$$

so that $y\left(t, t_{0}, y_{0}\right) \equiv y_{0}$ and

$$
x\left(t, t_{0}, x_{0}\right)=x_{0}\left[x_{0}^{2}\left(e^{-2 t}-e^{-2 t_{0}}\right)+1\right]^{-(1 / 2)} .
$$

Thus $x(t, 0,1)=e^{t}$ and $x(t, 0,-1)=-e^{t}$ which are not bounded in the future. In fact, the solution $x\left(t, t_{0}, x_{0}\right)$ is bounded in the future if and only if $\left|x_{0}\right|<\exp t_{0}$. Clearly $\mathrm{H}_{1}, \mathrm{H}_{2}$, and $\mathrm{H}_{3}$ hold. Let $y(t)$ be any solution of (6). If $|y(0)|<1$, then the solution $x\left(t, t_{0}, x_{0}\right)$ of (7) is bounded in the future if $t_{0} \geqq 0$ and $\left|x_{0}-y\left(t_{0}\right)\right|<1-|y(0)|$; that is, we could choose $T=0$ and $\delta=1-|y(0)|>0$. If $|y(0)| \geqq 1$, then $x\left(t, t_{0}, x_{0}\right)$ is bounded in the future if $t_{0}>\log |y(0)|$ and $\left|x_{0}-y\left(t_{0}\right)\right|$ $<\exp t_{0}-|y(0)|$; that is, we could choose $T=\log |y(0)|+1>0$ and $\delta=|y(0)|(e-1)>0$. However, if $|y(0)| \geqq 1$, then no such $\delta>0$ exists for $t_{0} \leqq \log |y(0)|$.

Proof of Theorem 1. Fundamental to this proof is the following nonlinear analog of the "variation of constants" formula: for any $\left(t_{0}, x_{0}\right) \in D$, let $y\left(t, t_{0}, x_{0}\right)$ and $x\left(t, t_{0}, x_{0}\right)$ be the corresponding solutions of (1) and (2). Then for as long as they both exist,

$$
x\left(t, t_{0}, x_{0}\right)=y\left(t, t_{0}, x_{0}\right)
$$

$$
+\int_{t_{0}}^{\imath} R\left(t, s, x\left(s, t_{0}, x_{0}\right)\right) g\left(s, x\left(s, t_{0}, x_{0}\right)\right) d s .
$$

(I believe this is due to Alekseev [3] and it can be proved by computing $(d / d s) y\left(t, s, x\left(s, t_{0}, x_{0}\right)\right)$ and integrating from $t_{0}$ to $t$.)

Let $y(t)$ be any solution of (1). By Remark 3 above, $y(t)$ is bounded in the future. Let $\bar{\gamma}$ be any point in its maximal interval. Then there is a constant $M$ such that $|y(t)| \leqq M$ on $[\bar{\gamma}, \infty)$. Take $\epsilon=1$; choose 
a corresponding $\delta$ by the uniform stability of $y(t)$. Choose $N=N(M+2)$ $>0$ by $\mathrm{H}_{2}$. Choose $T \geqq \bar{\gamma}$ so large that

$$
\int_{T}^{\infty} h_{M+2}(s) d s<N^{-1} .
$$

(Note that $T$ and $\delta$ both depend on $y(t)$, in general.) Let $t_{0} \geqq T$ and $\left|y\left(t_{0}\right)-x_{0}\right|<\delta$. Then $\left|y\left(t, t_{0}, x_{0}\right)\right| \leqq M+1$ on $\left[t_{0}, \infty\right)$. For as long as $\left|x\left(t, t_{0}, x_{0}\right)\right| \leqq M+2$ for $t \geqq t_{0}$, we have by (8)

$$
\begin{aligned}
\left|x\left(t, t_{0}, x_{0}\right)\right| & \leqq\left|y\left(t, t_{0}, x_{0}\right)\right|+\int_{t_{0}}^{t}\left|R\left(t, s, x\left(s, t_{0}, x_{0}\right)\right) g\left(s, x\left(s, t_{0}, x_{0}\right)\right)\right| d s \\
& \leqq M+1+\int_{t_{0}}^{t} N h_{M+2}(s) d s \\
& \leqq M+1+N \int_{T}^{\infty} h_{M+2}(s) d s<M+2 .
\end{aligned}
$$

Thus $\left|x\left(t, t_{0}, x_{0}\right)\right|<M+2$ on $\left[t_{0}, \infty\right)$, proving (a).

To prove (b), let $x(t)$ be any solution of (2), defined on some maximal interval $(\alpha, \infty)$, which is bounded in the future (the proof when $x(t)$ is defined on $[0, \infty)$ is even easier). Let $\gamma>\alpha$. Then there is a constant $M$ such that $|x(t)| \leqq M$ on $[\gamma, \infty)$. Let $\epsilon>0$. Choose $N=N(M)$ and $N_{1}=N(M+\epsilon)$ by $\mathrm{H}_{2}$. Then choose $T>0$ such that

$$
\int_{T}^{\infty} h_{M}(s) d s<\frac{\epsilon}{3 N} \text { and } \int_{T}^{\infty} h_{M+\epsilon}(s)<\frac{\epsilon}{3 N_{1}} .
$$

Let $t_{0} \geqq T$ and $x_{0}=x\left(t_{0}\right)$. (Incidentally, note that (8) immediately implies

$$
\left|x\left(t, t_{0}, x_{0}\right)-y\left(t, t_{0}, x_{0}\right)\right| \leqq N \int_{t_{0}}^{t} h_{M}(s) d s<\frac{\epsilon}{3}
$$

which shows that all solutions of (2) which are bounded in the future are close to certain solutions of (1) uniformly in $t$ for all large enough $t$. This is a type of asymptotic equivalence result which was also obtained by Onuchic for $\left(1^{\circ}\right)$ and $\left(2^{\circ}\right)$ in [1].) Choose $\delta=\delta\left(\epsilon / 3, y\left(t, t_{0}, x_{0}\right)\right)$ $>0$ by the uniform stability of $y\left(t, t_{0}, x_{0}\right)$. Thus $\delta$ depends only on $\epsilon$ and $t_{0}$. Let $\left|x_{0}-\bar{x}_{0}\right|<\delta$. Then

$$
\left|y\left(t, t_{0}, \bar{x}_{0}\right)-y\left(t, t_{0}, x_{0}\right)\right|<\frac{\epsilon}{3}
$$


for all $t \geqq t_{0}$. Also, for as long as $\left|x\left(t, t_{0}, \bar{x}_{0}\right)\right|<M+\epsilon$ for $t \geqq t_{0}$,

$$
\begin{aligned}
\left|x\left(t, t_{0}, \bar{x}_{0}\right)-x\left(t, t_{0}, x_{0}\right)\right| \\
\quad \leqq\left|y\left(t, t_{0}, \bar{x}_{0}\right)-y\left(t, t_{0}, x_{0}\right)\right|+\int_{t_{0}}^{t}\left[N_{1} h_{M+\epsilon}(s)+N h_{M}(s)\right] d s \\
\quad<\frac{\epsilon}{3}+\frac{\epsilon}{3}+\frac{\epsilon}{3}=\epsilon .
\end{aligned}
$$

Thus $\left|x\left(t, t_{0}, \bar{x}_{0}\right)\right|<M+\epsilon$ on $\left[t_{0}, \infty\right)$, hence (9) holds on $\left[t_{0}, \infty\right)$. We must now find $\delta$ for $t_{0}<T$. Let $\delta_{1}=\delta(\epsilon, T)$ as just obtained. If $\gamma \leqq t_{0}<T$, choose $\delta=\delta\left(\delta_{1}, t_{0}, T\right)=\delta\left(\epsilon, t_{0}\right)>0$ so that if $\left|\bar{x}_{0}-x_{0}\right|<\delta$, then

$$
\left|x\left(t, t_{0}, \bar{x}_{0}\right)-x\left(t, t_{0}, x_{0}\right)\right|<\min \left(\epsilon, \delta_{1}\right)
$$

for all $t_{0} \leqq t \leqq T$ by continuous dependence. Therefore $\mid x\left(T, t_{0}, \bar{x}_{0}\right)$ $-x\left(T, t_{0}, x_{0}\right) \mid<\delta_{1}$ so that (9) implies $\left|x\left(t, t_{0}, \bar{x}_{0}\right)-x\left(t, t_{0}, x_{0}\right)\right|<\epsilon$ on $[T, \infty)$. Thus $\left|x\left(t, t_{0}, \bar{x}_{0}\right)-x\left(t, t_{0}, x_{0}\right)\right|<\epsilon$ on $\left[t_{0}, \infty\right)$.

We have thus produced $\delta\left(\epsilon, t_{0}\right)$ for this $\epsilon$ and any $t_{0} \geqq \gamma$. Since $\epsilon$ is arbitrary, $x(t)$ is stable on $[\gamma, \infty)$. Since $\gamma$ is arbitrary, $x(t)$ is stable on $(\alpha, \infty)$, completing the proof.

\section{REFERENCES}

1. Nelson Onuchic, On the uniform stability of a perturbed linear system, J. Math. Anal. Appl. 6 (1963), 457-464.

2. L. Cesari, Asymptotic behavior and stability problems in ordinary differential equations, 2nd ed., Academic Press, New York, 1963.

3. V. M. Alekseev, An estimate for the perturbations of the solutions of ordinary differential equations, Vestnik Moskov. Univ. Ser. I Mat. Meh., 1961, no. 2, 28-36. (Russian)

UNIVERSITY OF MARYLAND 